\author{
Classification \\ Physics Abstracts \\ $1.680-7.130-7.420-7.460-7.480$
}

\title{
THERMODYNAMICS OF SMECTIC MIXTURES
}

\author{
F. C. FRANK $(*)$ \\ Laboratoire de Physique des Solides, Université Paris Sud, 91405 Orsay, France
}

(Reçu le 25 mars 1977, accepté le 21 avril 1977)

Résumé. - P. G. de Gennes discute le diagramme de phases thermodynamique d'un mélange de substances ayant des phases smectiques $\mathrm{A}$ et $\mathrm{C}$ à l'aide du développement de l'enthalpie libre suivant :

$$
F(T, x, \varphi)=F_{0}(T, x)+\frac{1}{2} a(T, x) \varphi^{2}+\frac{1}{4} b(T, x) \varphi^{4}+\cdots
$$

où $x$ est la fraction molaire du deuxième constituant et $\varphi$ le paramètre décrivant la brisure de symétrie (angle d'obliquité). Il conclut que la transition de phase est du second ordre si $b$ est positif lorsque $a(T, x)$ change de signe, la condition d'annulation de $a(T, x)$ définissant une limite de phase le long d'une ligne unique $x_{0}(T)$. Cette conclusion est erronée : la transition qui en découle est du premier ordre, à moins que ne soit aussi satisfaite la condition

$$
\partial^{2} F_{0} / \partial x^{2}>\frac{1}{2 b}\left(\frac{\mathrm{d} a}{\mathrm{~d} x}\right)^{2} .
$$

Si cette condition n'est pas satisfaite, il y a dans le diagramme un domaine de coexistence des phases A et $\mathrm{C}$, limité par des lignes de part et d'autre de $x_{0}(T)$, ce qui constitue une spinodale plutôt qu'une limite de phase.

Abstract. - P. G. de Gennes discusses the thermodynamic phase diagram of a mixture of substances generating smectic $\mathrm{A}$ and $\mathrm{C}$ phases with the aid of the expansion of free enthalpy :

$$
F(T, x, \varphi)=F_{0}(T, x)+\frac{1}{2} a(T, x) \varphi^{2}+\frac{1}{4} b(T, x) \varphi^{4}+\cdots
$$

where $x$ is mole fraction of second component, $\varphi$ the symmetry-breaking parameter (tilt angle), and concludes that the phase transition is one of second order, if $b$ is positive where $a(T, x)$ changes sign, which locates the single-line phase boundary $x_{0}(T)$. This is erroneous : the transition so arising is of first order unless in addition

$$
\partial^{2} F_{0} / \partial x^{2}>\frac{1}{2 b}\left(\frac{\mathrm{d} a}{\mathrm{~d} x}\right)^{2}
$$

and, without satisfaction of this condition there is a coexistence range in the phase diagram delimited by lines on either side of $x_{0}(T)$, which is a spinodal rather than a phase-boundary.

P. G. de Gennes (The Physics of Liquid Crystals (Clarendon Press), 1974, p. 277) treats the thermodynamics of the addition of a $\mathrm{C}$-smectogen to an $\mathrm{A}$ smectogen as follows. The free enthalpy $F$ is considered as a function of temperature, $T$, composition, $x$ (the mole-fraction of the second component) and a symmetry-breaking parameter $\varphi . \varphi$, identified in a model of the smectic phases as the tilt of the mole-

(*) Permanent address : The H. H. Wills Physics Laboratory, University of Bristol, Bristol 8, England. cules, is considered to be a continuously variable quantity, equal to 0 in the smectic-A phase, and $\neq 0$ in smectic C. Its most essential property is that an axis of infinite rotation symmetry in smectic-A ensures that $F(-\varphi)=F(\varphi)$. Hence odd terms are absent from the series development :

$$
\begin{aligned}
F(T, x, \varphi)=F_{0}(T, x)+ & \frac{1}{2} a(T, x) \varphi^{2}+ \\
& +\frac{1}{4} b(T, x) \varphi^{4}+\cdots
\end{aligned}
$$


At constant $x$ and $T$, this has stationary values at

$$
0=\partial F / \partial \varphi=a(T, x) \varphi+b(T, x) \varphi^{3}+\cdots
$$

which are minima or maxima according to the sign $(+$ or -$)$ of

$$
\partial^{2} F / \partial \varphi^{2}=a(T, x)+3 b(T, x) \varphi^{2}+\cdots .
$$

Thus, if $a>0$ there is a minimum at $\varphi=0$, making $\mathrm{A}$ the stable phase. If $a$ changes sign $\varphi=0$ becomes a maximum of $F$, and, provided $b>0$ there are now minima of $F$ ( $\varphi$ still being small) at

$$
\varphi= \pm(-a / b)^{1 / 2} \text {. }
$$

De Gennes deduces that when $b>0$ the phase change occurs as one of second order at the condition $a(T, x)=0$, represented by a single line in the $(x, T)$ phase diagram. On the other hand, when $b<0$, one must go to higher terms in (1) to find a minimum of $F$ which occurs at finite $\varphi$ giving rise to a first order transition with two lines in the $(x, T)$ phase diagram, between which the phases coexist.

This argument pays insufficient attention to the freedom of a two-component system to become inhomogeneous in composition, breaking into phases with greater and smaller values of $x$.

Let $F_{\varphi}(T, x)$ denote the value of $F$ minimized with respect to $\varphi$ at constant $x$ and $T$. Let us have $b>0$, and $a \gtrless 0$ according as $x \lessgtr x_{0}(T)$, so that $x_{0}(T)$ is de Gennes' 2nd order transition condition. Then

$$
\begin{aligned}
& x<x_{0}: F_{\varphi}(T, x)=F_{0}(T, x) \\
& x>x_{0}: F_{\varphi}(T, x)<F_{0}(T, x) .
\end{aligned}
$$

Close to $x_{0}$, so that $\varphi$ is small and we may neglect higher terms in (1), we have by use of (4) :

$$
x \sim x_{0}: F_{\varphi}(T, x)=F_{0}(T, x)-a^{2} / 4 b .
$$

Separation into phases of different composition will occur if, for some $x, \mathrm{~d}^{2} F_{\varphi} / \mathrm{d} x^{2}<0$. This condition may be brought about at higher $x$ and $\varphi$ by the effect of other terms but for $x$ just above $x_{0}$ (where $a$ is infinitesimal) we have :

$$
\mathrm{d}^{2} F_{\varphi} / \mathrm{d} x^{2}=\mathrm{d}^{2} F_{0} / \mathrm{d} x^{2}-\frac{1}{2 b}\left(\frac{\mathrm{d} a}{\mathrm{~d} x}\right)^{2}
$$

so that to de Gennes' conditions for a second order transition must be added another necessary condition

$$
\partial^{2} F_{0} / \partial x^{2}>\frac{1}{2 b}\left(\frac{\mathrm{d} a}{\mathrm{~d} x}\right)^{2} .
$$

If this is not satisfied, we need information about the other coefficients, and at other values of $x$ than $x_{0}(T)$ and its immediate neighbourhood : phases will coexist with compositions defined by a double tangent to $F_{\varphi}(x)$, one with $x<x_{0}$ and the other with $x$ above the range in which $\mathrm{d}^{2} F_{\varphi} / \mathrm{d} x^{2}<0 . \partial^{2} F_{\varphi} / \partial x^{2}$ changes sign at $x_{0}(t)$, since $\partial^{2} F_{0} / \partial x^{2}$ must be positive or else we should be involved with a different problem - phase separation independent of tilt. Thus $x_{0}(T)$ when (9) is not satisfied is the spinodal rather than the phase boundary.

First order smectic A-smectic $\mathrm{C}$ phase transitions of the kind indicated by de Gennes, as arising if $b<0$ when $a(T, x)$ changes sign, are a somewhat unrelated phenomenon. They signify that higher terms in (1) give rise at constant $T$ to minima of $F(T, x)$ occurring at finite $\varphi$ which for some $x$ (which is not necessarily that for the mixture whose stability we are testing) are lower than $F_{0}(T, x)$. Change of sign of $a(T, x)$ is not necessarily involved for any $x$. 